\title{
Validity and Intra Rater Reliability of a New Device for Tongue Force Measurement
}

\author{
Marta Carlota Diaz-Saez ${ }^{1,2}$, Hector Beltran-Alacreu ${ }^{2,3 *}$, Javier Gil-Castillo4, Gonzalo Navarro-Fernández ${ }^{1,2}$, \\ José Luis Cebrián Carretero ${ }^{5}$, Alfonso Gil-Martínez ${ }^{1,2,6}$
} \author{
(Spain) \\ ${ }^{2}$ CranioSPain Research Group. Centro Superior de Estudios Universitarios La Salle. 28023 Madrid (Spain) \\ ${ }^{3}$ Toledo Physiotherapy Research Group (GIFTO), Faculty of Physical Therapy and Nursing, Universidad de Castilla-La \\ Mancha. (Spain) \\ ${ }^{4}$ Neuro-Rehabilitation Group. Instituto Cajal. Consejo Superior de Investigación Científicas (CSIC) \\ (Spain) \\ ${ }^{5}$ Maxilofacial and Oral Surgery Department, Hospital Universitario La Paz, Madrid (Spain) \\ ${ }^{6}$ Unit of physiotherapy, Hospital La Paz Institute for Health Research (IdiPaz), Madrid (Spain)
}

${ }^{1}$ Physiotherapy Department, Centro Superior de Estudios Universitarios La Salle, Universidad Autónoma de Madrid, Madrid

Received 10 March 2021 | Accepted 23 December 2021 | Early Access 2 February 2022

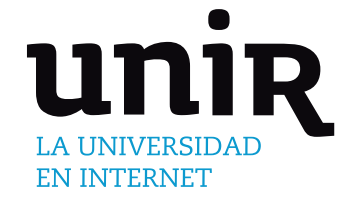

KEYWORDS

Device, Reliability, Temporomandibular Disorders, Tongue Force, Validity.

\begin{abstract}
Background. The tongue is made up of multiple muscles both extrinsic and intrinsic. The hyoid, jaw and maxillary complex contain the tongue, which hangs between these structures forming an important biomechanical system. This organ has to work in coordination with craniofacial structures to ensure normal orofacial functioning. There are different devices on the market for tongue force measurement. However, they are not accessible for patients due to their size and very high prices. Likewise, other devices have not yet carried out validity and reliability studies. The purpose of this study was to validate a new device proving that it is accurate compared to the algometer. Moreover, the study wanted to determine the intra-rater reliability of a protocol to assess the maximum tongue force in asymptomatic subjects. Material and methods. This is an observational-longitudinal study with repeated measurements. A prototype device was developed specifically for this study to measure tongue force through force-sensitive resistor sensors. The prototype system was equipped with a device to perform and transmit the measurement and a $\mathrm{C}++$ programming software in the computer to take data from the session. Different formulas were made to calibrate the system. For validity, the force measured by the prototype and the algometer was compared. For intra-rater reliability, 29 asymptomatic Spanish subjects were recruited, and a standardized protocol was carried out for the tests. Results. Experiments to assess validity showed a strong correlation $(r>0.97)$ and an excellent reliability $($ ICC $>0.90)$ between devices. On the other hand, the intra-rater reliability analysis showed an excellent ICC (0.93) with a 95\% CI of 0.86 to 0.97 and $\mathrm{a} \mathrm{MDC}_{90}$ of $6.26 \mathrm{~N}$. Conclusion. We demonstrated good validity values and high intra-rater reliability for the prototype device for the maximum tongue force.
\end{abstract}

\section{INTRODUCTION}

HE tongue is a muscle formed by extrinsic and intrinsic muscles
which are responsible for tongue movements and tongue shape
changes, respectively [1]. This organ belongs to the stomatognathic
system and has to work in coordination with other craniofacial
structures for the normal orofacial functions [2]. The hyoid, jaw and
maxillary complex contain the tongue, which hangs between these
structures forming an important biomechanical system [1], [2]. The
main functions the tongue participates in are swallowing, mastication

${ }^{*}$ Corresponding author

E-mail address: hector.beltran@uclm.es and speech, highlighting its important role during the oral and the pharyngeal phases of swallowing [3], [4]. In swallowing, the tongue has to generate a specific pressure to control and push the food bolus ensuring a well transportation from the oral cavity to the stomach. The applied force depends on the type of food bolus and must have to be coordinated with the jaw and hyoid movements [5]-[11]. During the tongue pressure against the anterior part of the hard palate, the hyolaryngeal complex has to elevate itself allowing a decline of the epiglottic cartilage to protect the airway. This coordinate process decreases the risk of suction increasing safety in the swallowing process. Therefore, any disturbance or pathology in one of these structures may result in a malfunctioning of the others. That is why, a reduction in tongue force could avoid the correct formation and placement of the food bolus. Moreover, a well propel and transport 
could be compromised with a drop in the tongue force. Likewise, the amount of waste accumulated in the oral and pharyngeal cavity could be increased and other symptoms associated with dysphagia can also be generated [5]-[11].

Different studies have shown changes in tongue force in orofacial pathologies, such as swallowing disorders, motor speech disorders, oromotor deficits or temporomandibular disorders [2], [12], [13]. Interest in studying tongue force in pathologies where these organ functions are limited or affected has led to the design of measuring systems with different force or pressure sensors. Some of these systems are dynamometers, miniaturized pressure transducers and electromyographic monitoring techniques. Their purpose is to offer an objective characterization [14].

In this study, a lower-cost system available for use at home to assess and train tongue force with different dynamics is presented. The importance of this device's implementation lies in its ability to reduce social and health care costs and waiting lists of the health system, as well as promoting patient independence at home. Because this prototype system is portable, handy and has an intuitive interface, it becomes a tool not only for professionals, but also for patients. All these things are very important to ensure home treatments based on exercises that offer patients feedback about their measurements. Moreover, these home training exercises may allow patients to maintain long-term effects of the therapy and avoid the overcrowding of health care services [15].

At this time, there are similar devices in the market which research has demonstrated to be useful in measuring tongue force in several scenarios [16]-[18]. However, these devices present some limitations when compared to the prototype of this study (Table I). This is because certain systems, such as the Iowa Oral Performance Instrument (IOPI), involve an expense of around 800-2000€, whereas the complete product presented in this study costs 90-100€ (estimated price) [19]. In addition, non-portable devices such as the Kay Swallowing Workstation (KSW) decreases the possibility of being accessible for patients [18]. Furthermore, the limited durability of the system components of other devices is another shortcoming that we try to eliminate in our system by using force-sensitive resistor (FSR) sensors. These sensors do not present mobile parts, which reduces the risk of breaking or wearing out. Moreover, they are not significantly affected by the noise or vibration and can operate between $-30^{\circ}$ and $70^{\circ}$ degrees [20]-[22] In addition, allergies have to be considered since they are intraoral measurements. The current literature does not mention tongue force devices made with hypoallergenic materials. Some studies show devices made with latex materials [23], while other researches do not provide information about the intraoral system's materials [3], [18], [19]. For this reason, we developed a device which provides a single- use hypoallergenic protection covering the FSR sensor like a sleeve made of nitrile.

Based on the above, the aim of the study is to validate the new device comparing the measurements to the algometer. The research also aims to determine the intra-rater reliability of a protocol to assess the maximum tongue force in asymptomatic subjects.

\section{METHODS}

This study is an observational-longitudinal study with repeated measurements. The research was approved by the ethical committee of the Centro Superior de Estudios Universitarios La Salle (CSEULSPI-036/2019). For the intra-rater reliability, asymptomatic individuals were recruited from the Hospital Universitario La Paz according to the Guidelines for Reporting Reliability and Agreement Studies (GRRAS) 2010 [24].

The research team was composed of a biomedical engineer and four experienced physical therapists. The biomedical engineer and two of the physical therapists (involved in the study) developed the measurement system for the validity, while the other physical therapists carried out the intra-rater reliability. Finally, all the data collected was analyzed by one of the assessors.

\section{A. Study Description}

\section{Validity}

Participants were not needed for the validity process. The biomedical engineer and two experienced physical therapists developed the measurements with the device and an algometer.

Wagner algometer (FPX25, Wagner Instruments, Greenwich CT, USA) was used in this study. The algometer is a device that can be used to standardize the intensity of palpation or to measure the degree of pressure used to evoke a painful response. It is a system that can be used to make kinesthetic measurements in an objective way. The algometer consists of a flat circular rubber tipped probe of $1 \mathrm{~cm}$ diameter attached to a soft-grip handle piston connected to a pressure sensitive strain gauge transducer, called a load cell. The force applied to the end of the probe is transmitted to the load cell and a voltage output is produced. This voltage output is directly related to the pressure exerted and allows for its calculation. Finally, the result is displayed on the LCD numerical display in lbf, Kgf, $\mathrm{N}$ or ozf. Furthermore, the device presents a calibration mechanism to measure and represent values in a graduate scale expressed as Newton or Kgf. These characteristics make the algometer suitable as comparison equipment.

TABle I. Comparison Between the Most Common Tongue Devices in the Market

\begin{tabular}{|c|c|c|c|c|}
\hline Item & New Device & IOPI & KSW & MOST \\
\hline System & $\begin{array}{c}\text { Computerized system with } 1 \\
\text { FSR sensor }\end{array}$ & Silicon air-filled bulb & $\begin{array}{c}\text { Computerized system with } 3 \\
\text { air-filled sensors }\end{array}$ & $\begin{array}{l}\text { Computerized system with } 4-5 \\
\text { sensors inside } 1 \text { intraoral piece }\end{array}$ \\
\hline $\begin{array}{l}\text { Stability of the } \\
\text { sensors }\end{array}$ & Good & Poor & Poor & Good \\
\hline Size & Small & Small & Large & Medium \\
\hline Portability & Portable & Portable & Non-portable & Portable \\
\hline Price & Cheap (90-100€) & Expensive (1.190€) & Unknown & Expensive (800-34.000€) \\
\hline Reliability & Yes & Yes & No & Yes \\
\hline Validity & Yes & No & No & No \\
\hline $\begin{array}{c}\text { Visualization } \\
\text { of signal }\end{array}$ & Excellent & Good & Excellent & Good \\
\hline Data integrity & Good & Poor-movement artefacts & Good-with fixed sensors & Good \\
\hline Patient usage & Hand held device & Hand held device & Fixed position & Fixed position \\
\hline
\end{tabular}




\section{Reliability}

A total of 32 asymptomatic Spanish subjects were recruited from the Hospital Universitario La Paz. Three subjects had to be excluded so a total of 29 asymptomatic participants were included in the study. The study was publicly advertised in different places so that people interested in the trial could participate. A non-randomized convenience sampling was performed for this study.

The whole procedure was developed from September 2019 to December 2019. The inclusion criteria were as follows: age between 18 and 65 years old; had not experienced any craniofacial/ temporomandibular/neck pain; had no facial palsy caused by a primary muscle disorder; and had no significant history of chronic pain disorder.

The exclusion criteria were: a surgery or history of traumatic injuries of the neck/head/face/tongue/teeth/jaw; cancer or an active infection of the neck/head/mouth; rheumatic disorders; neurological disorders; and pregnancy.

\section{B. Instrumentation}

For this study, a prototype device was specifically designed and developed to measure tongue force. The system consists of two main parts: device and software. The device is in charge of performing the measurement and transmission of the tongue force, while the software, located in the PC, allows taking data from the session (movement to be measured and time measured) and from patients (name, date of birth, sex and pathology) (Fig. 1). Moreover, the software shows tongue force in real time and stores the data corresponding to the session in an Excel database.

The device employs a piezo-resistive sensor to acquire force. This sensor, known as FSR sensor, is a type of technology which resists changes while a pressure is applied on the active surface. There is a negative correlation between resistance and force exerted. This means that the greater the force exerted, the lower the resistance that is generated [25]. For measurement of tongue force in the current study, a circular FSR sensor (Model: 402, Interlink Electronics, CA, USA) with $0.46 \mathrm{~mm}$ nominal thickness, an area of $12.7 \mathrm{~mm}$ diameter at the top and a measurement range of 0 to $10 \mathrm{~kg}$ was chosen [20]. This type of sensor has proven to be a good option compared to others on the market when dynamic measurements are required [22].

To measure the resistance of the FSR sensor, a voltage divider circuit was built with two resistors arranged in series, where one of them is the FSR [20]. The following formula was then applied to measure the FSR resistance (R_FSR):

$$
R_{F S R}=\frac{R}{V_{R}} \cdot\left(V-V_{R}\right)
$$

$V$ : input voltage, $V_{R}$ : resistance voltage, R: resistor. In our case, $\mathrm{V}=5 \mathrm{~V}$ and $\mathrm{R}=10 \mathrm{k} \Omega$

The resistance voltage was measured with a $\mathrm{C}++$ program developed in Arduino UNO, which uses an analog to digital converter (ADC) module of a 10-bit Atmega328P microcontroller to transform the analog signal to a digital signal whose values are between 0 and 1023 .

Since the variation of the resistance is not linear, it was decided to use conductance to calculate the force, as this allows interpretation on a linear scale. The conductance (C) is the inverse of the resistance, i.e.:

$$
C_{F S R}=1 / R_{F S R}
$$

Using the information provided in the sensor's own datasheet [20], an approximate calculation of the force was made by relating it to the conductance and dividing it into two force ranges (from 0 to $1 \mathrm{~kg}$ and from 1 to $10 \mathrm{~kg}$ ). For the small force range ( 0 to $1 \mathrm{~kg}$ ) the following linearized approximation of the force-conductance relationship was used:

$$
F_{F S R}[N]=C_{F S R} / 80
$$

Likewise, for the big force range (1 to $10 \mathrm{~kg}$ ), the following linearized approximation of the force-conductance relationship was used:

$$
F_{F S R}[N]=C_{F S R} / 30+1000 / 80
$$

This information is sent in real-time via USB communication to the PC, where it is displayed at the same time and it can be collected by the software developed in Java-based Processing. The interface has been designed and developed to be simple and intuitive for use in the clinical environment. This user-friendly interface, available for Windows, MacOS and Linux operating systems, facilitates the use of the system and the performance of specific measurements for a predetermined period of time by the professional for subsequent registration. In addition, it has been adapted for use on computers with different screen sizes. Currently, the movements enabled for recording in the interface are: lip to lip, tongue protrusion, tongue elevation, tongue depression, right tongue lateralization and left tongue lateralization. However, the system allows us to measure any type of movement, as long as the force is applied to the active surface of the sensor.

During measurements on subjects, a single-use hypoallergenic

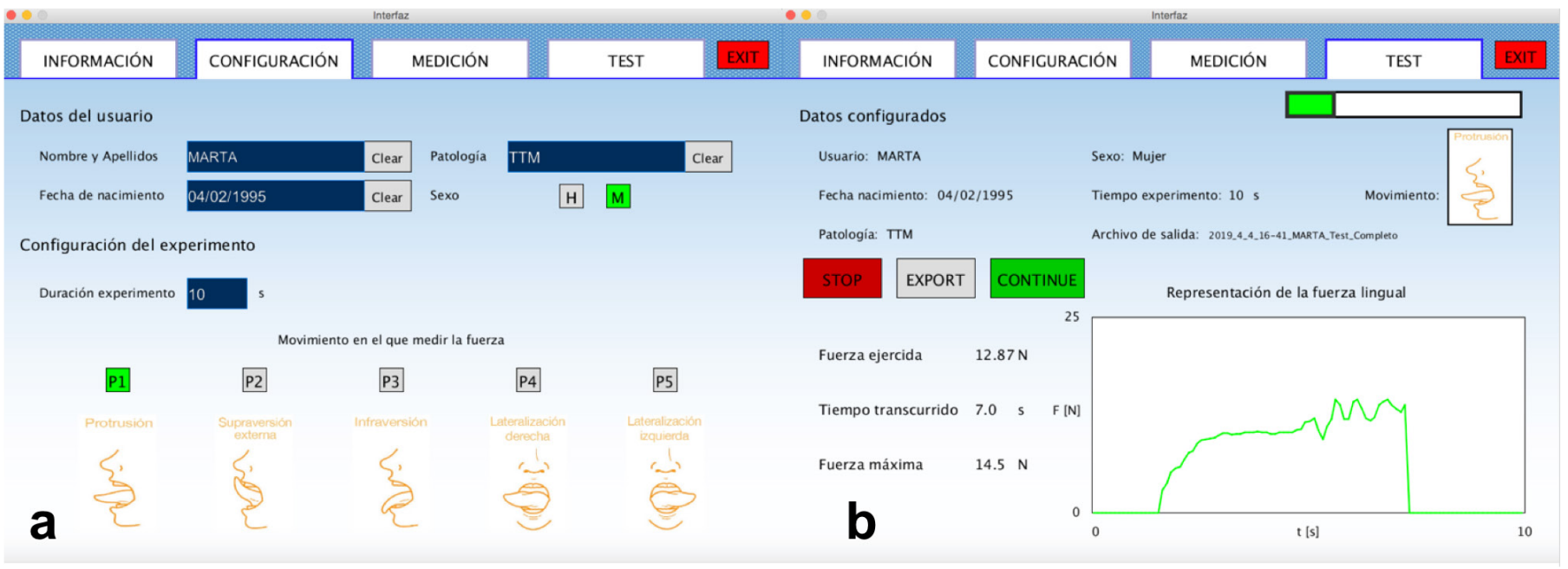

Fig. 1. Spanish prototype device interface view. a: Configuration screen view; b: Measurement screen view. 
protection made by nitrile is used to isolate the system from the patient so that the same system and sensor can be used between patients. In addition, this protection allows us to isolate the sensor from the temperature, which can be added by inserting it in the mouth to make the corresponding measurement. Due to this insulation, the temperature does not seem to be a parameter that can influence the measurement made.

\section{Procedure}

\section{Validity Procedure}

For the validation of the system, conditions were set-up in order to reproduce the environment in which the measurement was going to be performed. In addition, an algometer was used to validate the measurements that the system collected. For this purpose, the system remained fixed on a flat horizontal surface to maintain the position and avoid possible variations that can be introduced by an uneven surface. The sensor was covered by the hypoallergenic protection, as is carried out in measurements with patients. The purpose was to imitate the actual measuring environment and limit the influence of temperature. With the help of some markers on the table, the algometer was placed so that it was always in contact with the same point of the sensor and supported by the sensor's entire active surface. The perpendicularity between algometer and FSR sensor was maintained during the experiment with the help of a level and two vertical supports to which the algometer was fitted. The aim of this was to maintain repeatability during the whole experiment.

Once all the experimental conditions were configured, the measurement process started connecting the device and opening the software for 5 minutes to stabilize the whole system. Measurements corresponding to $2 \mathrm{~N}, 5 \mathrm{~N}, 10 \mathrm{~N}, 15 \mathrm{~N}, 20 \mathrm{~N}, 25 \mathrm{~N}, 30 \mathrm{~N}, 35 \mathrm{~N}, 40 \mathrm{~N}$ were obtained in the algometer for each FSR sensor. The adjustment to the desired value was made by gradually increasing the pressure, and independently for each measurement, for approximately 10 seconds. During the last 3 seconds a camera system was used to record the matching values of the measurement system and algometer and their subsequent analysis.

\section{Reliability Procedure}

Asymptomatic subjects were recruited for the reliability measurements. First of all, an informed consent was given to the participant. Secondly, they filled out some sociodemographic data and questions to know if they fulfilled the inclusion criteria. They were then asked about their initial subjective perception of fatigue before starting the measurements to ensure the subject started without it, and the assessor checked the proper functioning of the system. This variable was measured using a numeric verbal fatigue scale that consists on asking subjects quantify their perception of fatigue between 0 ("no fatigue") and 10 ("maximum fatigue conceivable") [26]. After that, participants sat down in front of the device and put a singleuse hypoallergenic protection covering the sensor like a sleeve made of nitrile. They had to protrude the tongue pressing the sensor with the tip of their tongue against the anterior part of the hard palate (Fig. 2) for 10 seconds with a verbal feedback from the assessor. This allows to assess tongue fatigue. They rested for 5 minutes and were again asked about their subjective perception of fatigue to ensure the subject performed the measurement without it. Finally, they repeated the measurement again trying to replicate the way the first measurement was conducted. All measurements were collected in a specific results paper for each subject, which were kept by the principal investigator.

\section{Sample Size Calculation}

Sample size was calculated using the method based on the Intraclass Correlation Coefficient (ICC) [27], [28]. Based on the ICC obtained

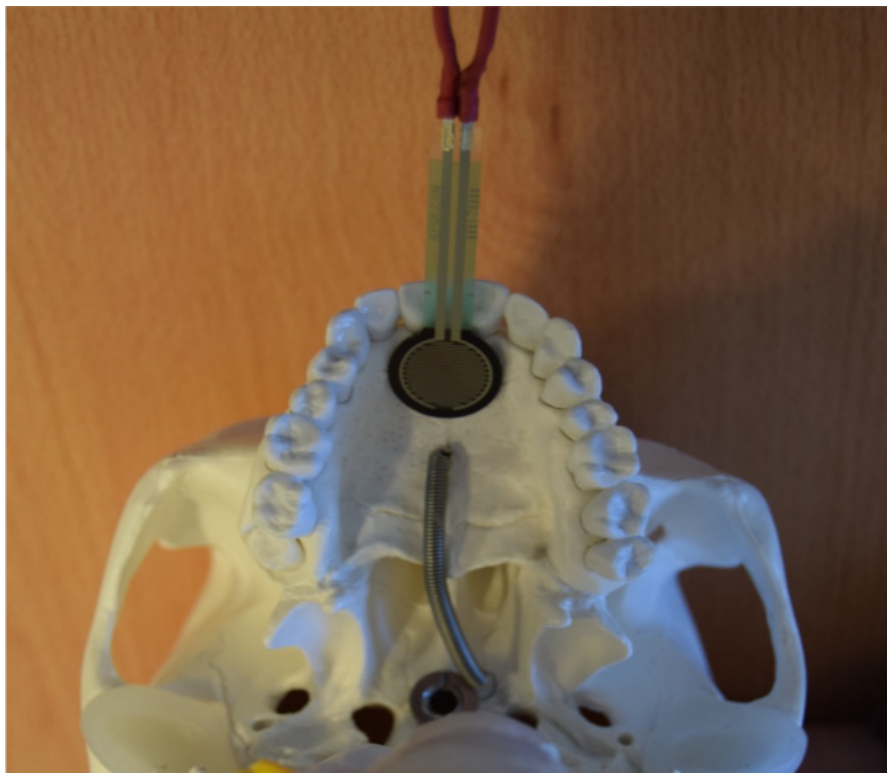

Fig. 2. Force pressing tongue against the superior part of the hard palate.

with the first 10 subjects of the present study, it was estimated that our ICC under the alternative hypothesis was 0.90 . A sample of 26 with 2 observations per subject was needed to achieve $80 \%$ power to detect an ICC of 0.90 , when the ICC under our null hypothesis was 0.75 using an F-test with a significance level of 0.05 . The final sample size was 29 to allow for possible dropouts $(10 \%)$.

\section{E. Statistical Analysis}

\section{Experiment to Assess the Validity of the Device}

Some measures were made simultaneously to compare the algometer and our device with two different FSR sensors. Those measures were made in some specific values of the algometer: $2 \mathrm{~N}, 5 \mathrm{~N}, 10 \mathrm{~N}, 15 \mathrm{~N}$, $20 \mathrm{~N}, 25 \mathrm{~N}, 30 \mathrm{~N}, 35 \mathrm{~N}$ and $40 \mathrm{~N}$. Thus, a total of 9 measurements for each FSR sensor were made. Those sensors will be named FSR-A and FSR-B to make it easier for the reader to understand the experiments. Two tests were made: the first one comparing algometry with FSR-A and the second one comparing algometry with FSR-B. Secondarily, a third comparison was made combining the data of test 1 and test 2 . All tests included the Shapiro-Wilk test to assess normal distribution of the data, Pearson's Correlation test to analyze algometry-device correlation, the Student's t test to assess algometry-device mean differences, and the intraclass correlation coefficient $\left(\mathrm{ICC}_{3,1}\right)$ to assess agreement between devices. Pearson's correlation test values above 0.60 were considered a strong correlation [29]. Finally, another ICC $_{3,1}$ was made to assess reliability between FSR-A and FSR-B.

\section{Reliability Analysis}

A total of $5 \mathrm{ICC}_{3,1}$ were calculated using SPSS statistical software (SPSS, Inc, Chicago, IL, USA): 4 included in the validity experiment and 1 to assess intra-rater reliability with healthy volunteers. The $\mathrm{ICC}_{3,1}$ was designated as the two-way analysis of variance mixed model for absolute agreement of single measures. Interpretation of the ICC was performed according to previously published categories: $<0.50$ is poor agreement, $0.50-0.75$ is moderate agreement, and $>0.75$ is good to excellent agreement [30].

Bland-Altman plots were constructed only for intra-rater reliability using mean differences between measurements [31]. Limits of agreement (LOA) were calculated as mean differences \pm (standard deviation multiplied by 1.96) [32]. Calculation of the occurrence of systematic or random changes in the data means that it was performed 
TABLE II.A. Pearson's Correlation and Student's T Test for Validity Tests (Newtons)

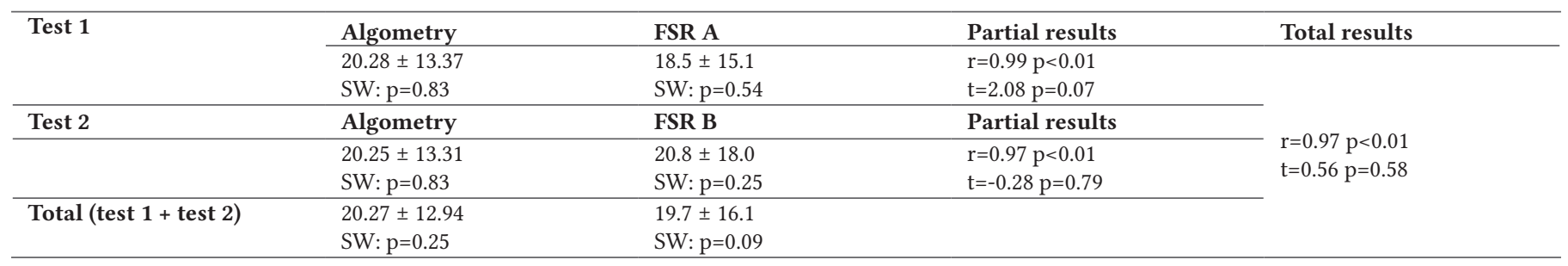

TABLE II.B. Between-Device and FSR A- FSR B Reliability Tests (Newtons)

\begin{tabular}{lllll}
\hline Measure & Mean difference & ICC $(\mathbf{9 5 \%}$ CI $)$ & SEM & MDC90 \\
\hline Algometry - FSR A & 1.77 & $0.98(0.88$ to 1.00$)$ & 1.96 & 4.57 \\
\hline Algometry - FSR B & -0.55 & $0.94(0.75$ to 0.99$)$ & 3.76 & 8.78 \\
\hline Algometry - FSR total & 0.61 & $0.95(0.88$ to 0.98$)$ & 3.23 & 7.53 \\
\hline FSR A - FSR B & 0.45 & $0.97(0.85$ to 0.99$)$ & 2.80 & 6.53 \\
\hline
\end{tabular}

Results are showed as mean \pm standard deviation.

SW: Shapiro-Wilk test; ICC: intraclass correlation coefficient; CI: confidence interval

SEM: standard error of measurement; MDC: minimal detectable change; FSR: force-sensitive resistor.

TABLE III. Intra-Rater Reliability Analysis and Bland-Altman Plot (Newtons)

\begin{tabular}{ll}
\hline Reliability Analysis & \\
\hline Measure 1 & $19.89 \pm 9.59^{\mathrm{a}}$ \\
Measure 2 & $19.44 \pm 10.82^{\mathrm{a}}$ \\
ICC (95\% CI) & $0.93(0.86$ to 0.97$)$ \\
SEM & 2.68 \\
MDC90 & 6.26 \\
\hline Bland-Altman Plot & $0.45 \pm 3.79^{\mathrm{a}}$ \\
\hline Mean difference & -0.93 to $1.83^{\mathrm{b}}$ \\
95\% CI mean difference & -6.98 to 7.88 \\
\hline Limits of Agreement & \\
\hline
\end{tabular}

${ }^{\text {a }}$ data showed as mean \pm standard deviation; ${ }^{b} 0$ is included in the $\mathrm{CI}(\mathrm{p}>0.05)$

ICC: intraclass correlation coefficient; CI: confidence interval; SEM: standard error of measurement; MDC: minimal detectable change.

through a calculation of $95 \%$ confidence intervals of the mean differences between the values of the measurements.

Measurement error was expressed as SEM. The SEM was calculated as $\sqrt{(R M S)} \times \sqrt{(1-I C C)}$, with RMS being the root mean square [30].

Responsiveness was determined with minimal detectable change at $90 \%$, which was calculated as SEM x $1.65 \times \sqrt{2}$ [33], [34]. The $\mathrm{MDC}_{90}$ expresses the minimal change required to be $90 \%$ confident that the change observed between two measurements reflects a real change and not a measurement error.

\section{RESUlTS}

\section{A. Experiments to Assess the Validity of the Device}

All measures showed normal distribution in the Shapiro Wilk test ( $p>0.05)$. Both partial and total results showed a strong correlation between devices $(r>0.97)$ and no statistically significant mean differences $(p>0.05)$. In addition, between-device reliability analysis and reliability analysis for both FSR measures showed an ICC $>0.90$. The results of all the experiments are summarized in Tables II.a and II.b.

\section{B. Intra-rater Reliability}

A total of 32 subjects were recruited for eligibility. Of those, 3 subjects did not meet the inclusion criteria because they had temporomandibular joint disorder diagnosed. Finally, 29 healthy subjects were included in the reliability analysis (17 men and 12 women aged $40.18 \pm 14.99)$. The Shapiro-Wilk test showed normal distribution of the data $(\mathrm{p}>0.05)$. Descriptive data, $\mathrm{ICC}_{3,1}, \mathrm{SEM}, \mathrm{MDC}_{90}$ and the Bland-Altman analysis with $95 \% \mathrm{CI}$ and LOA are summarized in Table III. The ICC obtained was excellent (ICC $=0.93$ ), with a $95 \%$ $\mathrm{CI}$ of 0.86 to 0.97 . Graphical representations of the Bland-Altman plot are shown in Fig. 3.

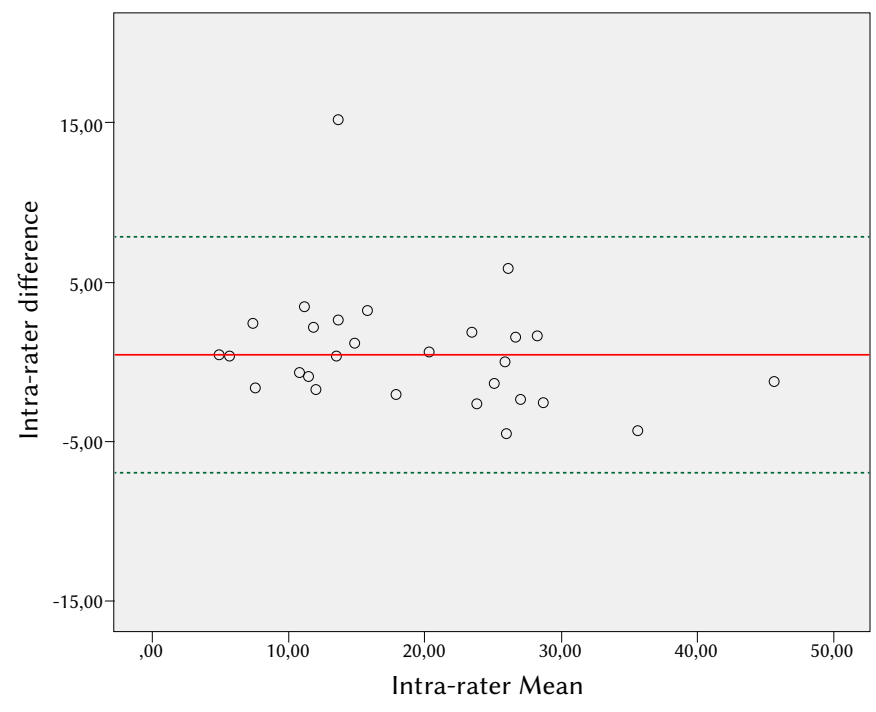

Fig. 3. Bland-Altman plot. 
IV. Discussion

The main objective of the present study was to verify the validity and intra-rater reliability of a new device for the measurement of maximum tongue force using an FSR sensor. To the authors' knowledge, this is the first validity and intra-rater reliability study of a device that uses an FSR sensor to measure tongue force.

At present, there is no test or device that has become the gold standard for measuring tongue force [35]. Although the IOPI is the most studied and used device for assessing tongue force [16], [17], [36], [37], it still does not have a validity study. In addition to the IOPI, there are different devices to measure tongue force, such as the Madison Oral Strengthening Therapeutic (MOST) [38] and the KSW [39], but they have not conducted validity studies either. For this reason, we do not have enough data to compare our validity results with other tongue force measurement devices. However, we know that the Interlink 402 FSR sensors used in this research have demonstrated great precision in force measurements for dynamic exercises against other FSR sensors [22]. FSR sensors have shown a reliability of the 93\% for bite force measure in several dentistry studies [40]. Moreover, Ahmed et al. observed an accuracy of over $95 \%$ in dynamic exercises for FSR sensors based on previous results [22], [25]. These sensors are characterized by their easy integration, their small size, their low weight and their low cost. All of this combined with the existing scientific literature supporting their reliability are the main reasons they are chosen for noninvasive force measurements [22], [41]-[43].

The Adam et al. study showed a good intra-rater reliability for the IOPI with high or very high ICC (0.77-0.90) in asymptomatic subjects $(n=51)$ with an average age of $28.2 \pm 9.3$ [16]. Further, White et al. reported an excellent intra-rater reliability (ICC 0.92) for the KSW, with a 95\% IC (0.85-0.97) in asymptomatic subjects with an average age of $64.7 \pm 10.2$ [3]. From our perspective, the device presented in this research seems to be a very good choice since the intra-rater reliability is slightly higher than the IOPI and very similar to the KSW. Its advantage over the KSW is its greater portability. There are few studies in which standard values of tongue pressure measurements were considered [44], [45]. In all those studies, the IOPI device or an IOPI-like design device was used. For example, Utanohara et al. showed an average maximum tongue pressure between $41.7 \mathrm{kPa}$ (kilopascal) in young subjects to $31.9 \mathrm{kPa}$ in old subjects, using a disposable tongue pressure measurement device with an IOPI-like design [44]. Also, Crow et al. carried out a similar experiment using IOPI and showed similar results (66.7 to $74.8 \mathrm{kPa}$ ) [45]. Note that both devices expressed results in pressure units $(\mathrm{kPa})$ while our new device expressed results in force units (Newtons) so we cannot directly compare results. If our results were transformed to pressure units, it could be obtained, approximately, $155.5 \mathrm{kPa}$ as maximum tongue force. This difference could be justified because the measurement protocols of the IOPI and our sensor are different. Crow et al. mentioned a protocol of measurement [46] in which it can be observed that raising the tongue and compressing the bulb of the IOPI onto the hard palate is not the same movement than making a protrusion of the tongue against the posterior side of superior incisor and palate with the tip of the tongue.

Regarding the SEM and $\mathrm{MDC}_{90}$ values, the results for the studied device were relatively low. It has to be considered that the way the SEM is calculated may differ between studies. Adams et al. used the standard deviation and our research used the RMS [16]. The RMS has been considered an alternative way to calculate the SEM, avoiding the uncertainties generated by the selection of the ICC type [30]. However, the low results for the SEM and the $\mathrm{MDC}_{90}$ indicates an agreement between studies in spite of the differences mentioned.

The available scientific evidence examines other devices that measure tongue force, such as the MOST or ad hoc devices, some of which have been compared with and formed partnerships with the IOPI and the KSW [18]. Nevertheless, there is an important limitation since these other devices have not yet carried out validity and reliability studies [18], [23], [47], [48]. For this reason, we cannot compare our results with these ad hoc devices. However, we can point out the existing differences between the devices on the market and the device used in this research. First of all, many of them have a limited durability because of the components used. Secondly, they have very high prices, poor stability systems or sensors that keep the mouth open, which affects the position of the different orofacial structures and hence tongue position [23], [48]-[50]. In contrast, the studied device is cheaper (90-100€), brings more stability due to the smooth sensor surface, and its small size avoids affecting the anatomical systems. Moreover, the device of this study uses hypoallergenic materials while the others do not, and this may be an important consideration when working with intraoral measurements [23], [48]-[50].

Finally, it should be highlighted that the main purpose of these devices is to quantify maximum tongue force of the different movements and tongue pressure. In addition, they may help in the diagnosis and strengthening of the tongue muscles. As a result, reliability studies like those developed by the IOPI, the KSW and the device proposed in this research are needed. Furthermore, validation of all devices is required in order to support their clinical use, as has been carried out in this study. These studies are needed in order to compare devices and select the best option.

\section{A. Clinical Implications}

From a clinical point of view, having validated instruments proves to be of great value. In this case, the instrument is a tongue force resistance device that could have an important positive impact on the treatment of patients with tongue disorders. Some of these primary tongue disorders could be orofacial cancer, swallowing disorders or disturbances associated with dysarthria. Other secondary tongue disorders may also benefit from this device, such as temporomandibular disorders. This is because some research has observed significant changes in tongue force in patients with this pathology [12], [13]. In this study, we assessed the protrusion movement of the tongue against anterior part of hard palate. That movement could be done by the action of the genioglossus, a midline muscle that can influence tongue shape and protract the tongue body [51]. This muscle has been identified as one of the core muscles of the tongue [52] and it is related with both functions: speech and swallowing [52], [53]. That is why, we consider that this research has important clinical implications because this new device can be used to assess objectively protraction movement of the tongue, but also it could be used as a training tool for improving speech and swallowing.

On the other hand, it should be noted that the 1 maximum repetition (1MR) system, a recognized system for assessing muscle strength, cannot be applied to an organ like the tongue. Moreover, this apparatus allows us to calculate the desired percentage through the force peak, based on the value established by it [36]. Although the subjects of this study did not see the screen while they were doing the force, it would also enable patients and professionals to develop force and resistance training with the visual biofeedback the system includes.

Moreover, future research lines should assess the utility and effectiveness of this device in tele rehabilitation processes. These are currently booming, and the device presented in this study could increase the quality of patient's assessment and monitoring in a home treatment.

Finally, an important point to be considered is the need to make technology available to as wide a population as possible. Having access to the device will allow professionals to get objective measurements in the clinic for giving specific exercise doses. This also, will promote 
patient independence at home controlling the training and observing the progression. For this reason, a key component of the healthcare system is to develop reliable and useful tools with lower prices like this device. Likewise, the portability and intuitive interface of this prototype system makes it a tool which could reduce social and health care costs and waiting lists of the health system.

Future studies need to be developed to know the inter-rater reliability and determine normality values in asymptomatic subjects and temporomandibular or speak disorder patients. The authors of this work also recommend planning quality clinical trials to verify the effects of different types of tongue exercises in patients with tongue disturbance pathologies.

\section{B. Limitations}

The present study has some limitations. First, convenience sampling was used due to the impossibility of accessing big databases for a simple random sampling.

Second, all the participants were asymptomatic, so statistical results have to be carefully considered when used in populations with pathology.

Third, this study has not considered the sensor deformation inside the mouth of the patients while tongue pressure is taking place. We recognize the need to work on future improvement of the device, adding a further element that eliminates the deformation of the sensor during tongue pressure. Moreover, this improvement needs to consider adding a sensitive feedback, as a grooved part, in order to facilitate a specific tongue pressure point for the patient.

Fourth, another current limitation is that the sensor can be damaged due to intra-oral misuse. Although sensor damage is complicated due to it resists millions of measurements without altering its operation, it could be damage if patients bite the sensor. That is why, work is underway to isolate and protect this sensor in order to solve this type of problem in the future. Nevertheless, the device has been designed in such a way that the replacement of these sensors can be done easily. In addition, they are very cheap sensors (4-7€).

\section{CONCLUSION}

The data obtained in this study suggest that this new device can be used by clinicians due to the good validity values and high reliability shown $($ ICC $=0.93)$ for intra-rater evaluation of the maximum tongue force. It allows to obtain objective measurements of tongue force in clinical practice in order to help clinicians with the diagnosis process and treatment progression. This device also has a low economic cost, is easy to handle and has various utilities, making this tool a great alternative in the measurement of tongue force-endurance due to the few ones that are available in the market have these limitations.

\section{ACKNOWLEDGEMENTS}

The authors would like to thank the Centro Superior of Estudios Universitarios La Salle for funding the English language edition of this manuscript. Moreover, we thank the collaboration of Pablo Jordi Perea, the speech language therapist in the Functional Rehabilitation Institute of the Centro Superior of Estudios Universitarios La Salle. HBA would like to thank the European Regional Development Fund (2020/5154).

\section{FUNDING}

Centro Superior de Estudios Universitarios La Salle funded the English-language edition of this manuscript.
Beltran-Alacreu $\mathrm{H}$ is financed by the European Regional Development Fund (2020/5154).

\section{CONFLICT OF INTEREST}

No conflict of interest was declared by the authors of this study.

\section{ETHICS}

The experiments carried out in this study complied with ethics Committee of Centro Superior de Estudios Universitarios La Salle (CSEULS). Registration number: CSEULS-PI-036/2019.

\section{REFERENCES}

[1] W. Wang, C. Di, S. Mona, L. Wang, and M. Hans, "Tongue Function: An Underrecognized Component in the Treatment of Obstructive Sleep Apnea with Mandibular Repositioning Appliance," Canadian Respiratory Fournal, vol. 2018, pp. 1-7, Nov. 2018, doi: 10.1155/2018/2157974.

[2] G. Messina, "The Tongue, Mandible, Hyoid System," European journal of translational myology, vol. 27, no. 1, p. 6363, Feb. 2017, doi: https://dx.doi. org/10.4081/ejtm.2017.6363.

[3] R. White, S. M. Cotton, J. Hind, J. Robbins, and A. Perry, "A Comparison of the Reliability and Stability of Oro-lingual Swallowing Pressures in Patients with Head and Neck Cancer and Healthy Adults," Dysphagia, vol. 24, no. 2, pp. 137-144, Jun. 2009, doi: 10.1007/s00455-008-9181-0.

[4] H. Yamanashi et al., "Validity of maximum isometric tongue pressure as a screening test for physical frailty: Cross-sectional study of Japanese community-dwelling older adults," Geriatrics \& Gerontology International, vol. 18, no. 2, pp. 240-249, Feb. 2018, doi: 10.1111/ggi.13166.

[5] M. A. Nicosia et al., "Age Effects on the Temporal Evolution of Isometric and Swallowing Pressure," The fournals of Gerontology Series A: Biological Sciences and Medical Sciences, vol. 55, no. 11, pp. M634-M640, Nov. 2000, doi: 10.1093/gerona/55.11.M634.

[6] H. Taniguchi et al., "Fluoroscopic Evaluation of Tongue and Jaw Movements During Mastication in Healthy Humans," Dysphagia, vol. 28, no. 3, pp. 419-427, Sep. 2013, doi: 10.1007/s00455-013-9453-1.

[7] J. Kieser, M. Farland, H. Jack, M. Farella, Y. Wang, and O. Rohrle, "The role of oral soft tissues in swallowing function: what can tongue pressure tell us?," Australian Dental fournal, vol. 59, pp. 155-161, Jun. 2014, doi: 10.1111/adj.12103.

[8] M. Peladeau-Pigeon and C. M. Steele, "Age-Related Variability in Tongue Pressure Patterns for Maximum Isometric and Saliva Swallowing Tasks," Journal of Speech, Language, and Hearing Research, vol. 60, no. 11, pp. 3177-3184, Nov. 2017, doi: 10.1044/2017_JSLHR-S-16-0356.

[9] J. Furuya, S. Nakamura, T. Ono, and T. Suzuki, "Tongue pressure production while swallowing water and pudding and during dry swallow using a sensor sheet system," fournal of Oral Rehabilitation, vol. 39, no. 9, pp. 684-691, Sep. 2012, doi: 10.1111/j.1365-2842.2012.02319.x.

[10] P. Bourdiol, A. Mishellany-Dutour, M.-A. Peyron, and A. Woda, "Tonguemandible coupling movements during saliva swallowing," fournal of Oral Rehabilitation, vol. 41, no. 3, pp. 199-205, Mar. 2014, doi: 10.1111/ joor.12135.

[11] K. Hori et al., "Role of tongue pressure production in oropharyngeal swallow biomechanics," Physiological Reports, vol. 1, no. 6, p. e00167, Nov. 2013, doi: 10.1002/phy2.167.

[12] R. R. Rosa, M. da R. S. Bueno, R. R. Migliorucci, A. G. Brasolotto, K. F. Genaro, and G. Berretin-Felix, "Tongue function and swallowing in individuals with temporomandibular disorders," fournal of Applied Oral Science, vol. 28, 2020, doi: 10.1590/1678-7757-2019-0355.

[13] M. Corsalini, D. Di Venere, F. Pettini, D. Lauritano, and M. Petruzzi, "Temporomandibular Disorders in Burning Mouth Syndrome Patients: An Observational Study," International fournal of Medical Sciences, vol. 10, no. 12, pp. 1784-1789, 2013, doi: 10.7150/ijms.6327.

[14] S. N. Robinovitch, C. Hershler, and D. P. Romilly, "A tongue force measurement system for the assessment of oral-phase swallowing disorders," Archives of physical medicine and rehabilitation, vol. 72, no. 1, pp. 38-42, Jan. 1991. 
[15] K. Frändin et al., "Long-Term Effects of Individually Tailored Physical Training and Activity on Physical Function, Well-Being and Cognition in Scandinavian Nursing Home Residents: A Randomized Controlled Trial," Gerontology, vol. 62, no. 6, pp. 571-580, 2016, doi: 10.1159/000443611.

[16] V. Adams, B. Mathisen, S. Baines, C. Lazarus, and R. Callister, "Reliability of Measurements of Tongue and Hand Strength and Endurance Using the Iowa Oral Performance Instrument with Healthy Adults," Dysphagia, vol. 29, no. 1, pp. 83-95, Feb. 2014, doi: 10.1007/s00455-013-9486-5.

[17] V. Adams, B. Mathisen, S. Baines, C. Lazarus, and R. Callister, "A Systematic Review and Meta-analysis of Measurements of Tongue and Hand Strength and Endurance Using the Iowa Oral Performance Instrument (IOPI), Dysphagia, vol. 28, no. 3, pp. 350-369, Sep. 2013, doi: 10.1007/s00455-013-9451-3.

[18] M. Yoshikawa, M. Yoshida, K. Tsuga, Y. Akagawa, and M. E. Groher, "Comparison of three types of tongue pressure measurement devices," Dysphagia, vol. 26, no. 3, pp. 232-7, Sep. 2011, doi: 10.1007/s00455-0109291-3.

[19] "IOPI Medical LLC. Iowa Oral Performance Instrument: users manual," 2008.

[20] State-of-the-Art Pointing Solutions for the OEM, "Force Sensing Resistor Integration Guide and Evaluation Parts Catalog," Interlinks Electronics, 2002.

[21] " $F R^{\mathrm{TM}}$ Integration Guide Interlink Electronics FSR ${ }^{\mathrm{TM}}$ Force Sensing Resistors ${ }^{\mathrm{TM}} \mathrm{FSR}^{\circledast}$ Integration Guide."

[22] S. Parmar, I. Khodasevych, and O. Troynikov, "Evaluation of Flexible Force Sensors for Pressure Monitoring in Treatment of Chronic Venous Disorders," Sensors, vol. 17, no. 8, p. 1923, Aug. 2017, doi: 10.3390/ s17081923.

[23] Y. Utanohara, R. Hayashi, M. Yoshikawa, M. Yoshida, K. Tsuga, and Y. Akagawa, "Standard Values of Maximum Tongue Pressure Taken Using Newly Developed Disposable Tongue Pressure Measurement Device," Dysphagia, vol. 23, no. 3, pp. 286-290, Sep. 2008, doi: 10.1007/s00455-0079142-z.

[24] J. Kottner et al., "Guidelines for Reporting Reliability and Agreement Studies (GRRAS) Were Proposed," fournal of clinical epidemiology, vol. 64, no. 1, 2011, doi: 10.1016/J.JCLINEPI.2010.03.002.

[25] T. Ahmed, N. A. Rahman, and M. K. Alam, "Validation and reliability of a prototype orthodontic bracket debonding device equipped with force-sensitive resistor (FSR): a novel method of measuring orthodontic bracket debonding force in vivo," Prog Orthod, vol. 20, no. 1, p. 26, 2019, doi: 10.1186/s40510-019-0277-x.

[26] M. J. Hjermstad et al., "Studies comparing Numerical Rating Scales, Verbal Rating Scales, and Visual Analogue Scales for assessment of pain intensity in adults: a systematic literature review," fournal of pain and symptom management, vol. 41, no. 6, pp. 1073-93, Jun. 2011, doi: 10.1016/j.jpainsymman.2010.08.016.

[27] S. D. Walter, M. Eliasziw, and A. Donner, "Sample size and optimal designs for reliability studies," Statistics in Medicine, vol. 17, no. 1, pp. 101-110, Jan. 1998, doi: 10.1002/(sici)1097-0258(19980115)17:1<101::aid$\operatorname{sim} 727>3.0 . c 0 ; 2-\mathrm{e}$.

[28] M. Adam Bujang and N. Baharum, "A simplified guide to determination of sample size requirements for estimating the value of intraclass correlation coefficient: a review," Archives of Orofacial Sciences, vol. 12, no. 1, pp. 1-11, 2017.

[29] D. E. Hinkle, W. Wiersma, and S. G. Jurs, Applied statistics for the behavioral sciences. Des Moines, Iowa: Houghton Mifflin, 1988.

[30] J. P. Weir, "Quantifying Test-Retest Reliability Using the Intraclass Correlation Coefficient and the SEM," The fournal of Strength and Conditioning Research, vol. 19, no. 1, p. 231, Feb. 2005, doi: 10.1519/15184.1.

[31] C. Bunce, "Correlation, Agreement, and Bland-Altman Analysis: Statistical Analysis of Method Comparison Studies," American fournal of Ophthalmology, vol. 148, no. 1, pp. 4-6, Jul. 2009, doi: 10.1016/j. ajo.2008.09.032.

[32] J. M. Bland and D. G. Altman, "Comparing methods of measurement: why plotting difference against standard method is misleading," Lancet (London, England), vol. 346, no. 8982, pp. 1085-7, Oct. 1995, doi: 10.1016/ s0140-6736(95)91748-9.

[33] S. M. Haley and M. A. Fragala-Pinkham, "Interpreting Change Scores of Tests and Measures Used in Physical Therapy," Physical Therapy, vol. 86, no. 5, pp. 735-743, May 2006, doi: 10.1093/ptj/86.5.735.
[34] K. W. Wyrwich, "Minimal Important Difference Thresholds and the Standard Error of Measurement: Is There a Connection?", fournal of Biopharmaceutical Statistics, vol. 14, no. 1, pp. 97-110, Dec. 2004, doi: 10.1081/BIP-120028508.

[35] E. Versi, "'Gold standard' is an appropriate term," BMf: British Medical Journal, vol. 305, no. 6846, p. 187, 1992, doi: 10.1136/BMJ.305.6846.187-B.

[36] V. S. McKenna, B. Zhang, M. B. Haines, and L. N. Kelchner, "A Systematic Review of Isometric Lingual Strength-Training Programs in Adults With and Without Dysphagia," American fournal of Speech-Language Pathology, vol. 26, no. 2, pp. 524-539, May 2017, doi: 10.1044/2016_ AJSLP-15-0051.

[37] N. P. Solomon, H. M. Clark, M. J. Makashay, and L. A. Newman, "Assessment of orofacial strength in patients with dysarthria," fournal of Medical Speech-Language Pathology, vol. 16, no. 4, pp. 251-258, 2008.

[38] J. Ulrich Sommer, R. Birk, K. Hörmann, and B. A. Stuck, "Evaluation of the maximum isometric tongue force of healthy volunteers," European Archives of Oto-Rhino-Laryngology, vol. 271, no. 11, pp. 3077-3084, Nov. 2014, doi: 10.1007/s00405-014-3103-6.

[39] R. K. Balasubramaniam, S. Babu, M. A. Anil, A. L. Varghese, Z. R. Hussain, and D. F. Dsouza, "Does Tongue - Hold Maneuver Affect Respiratory Swallowing Coordination? Evidence from Healthy Adults," pp. 68-71, 2019, doi: 10.4103/jnsbm.JNSBM.

[40] C. Fernandes, P. Glantz, S. Svensson, and A. Bergmark, "A Novel Sensor for Bite Force Determinations," Dental materials: official publication of the Academy of Dental Materials, vol. 19, no. 2, 2003, doi: 10.1016/S01095641(02)00020-9.

[41] J. S. Schofield, K. R. Evans, J. S. Hebert, P. D. Marasco, and J. P. Carey, "The effect of biomechanical variables on force sensitive resistor error: Implications for calibration and improved accuracy," fournal of Biomechanics, vol. 49, no. 5, pp. 786-792, Mar. 2016, doi: 10.1016/j. jbiomech.2016.01.022.

[42] L. Paredes-Madrid, A. Matute, A. F. Cruz-Pacheco, C. A. Parra-Vargas, \& Elkin, and I. Gutiérrez-Velásquez, "Experimental characterization, modeling and compensation of hysteresis in force sensing resistors," Revista DYNA, vol. 85, no. 205, pp. 191-198, 2018, doi: 10.15446/dyna. v85n205.66432.

[43] R. Tarricone, A. Torbica, and M. Drummond, "Challenges in the Assessment of Medical Devices: The MedtecHTA Project," Health Economics, vol. 26, pp. 5-12, Feb. 2017, doi: 10.1002/hec.3469.

[44] Y. Utanohara, R. Hayashi, M. Yoshikawa, M. Yoshida, K. Tsuga, and Y. Akagawa, "Standard values of maximum tongue pressure taken using newly developed disposable tongue pressure measurement device," Dysphagia, vol. 23, no. 3, pp. 286-90, Sep. 2008, doi: 10.1007/s00455-0079142-z.

[45] H. C. Crow and J. A. Ship, "Tongue strength and endurance in different aged individuals," The journals of gerontology. Series A, Biological sciences and medical sciences, vol. 51, no. 5, pp. M247-50, Sep. 1996, doi: 10.1093/ gerona/51a.5.m247.

[46] D. A. Robin, A. Goel, L. B. Somodi, and E. S. Luschei, "Tongue strength and endurance: Relation to highly skilled movements," fournal of Speech and Hearing Research, vol. 35, no. 6, pp. 1239-1245, 1992, doi: 10.1044/ jshr.3506.1239.

[47] R. Hayashi, K. Tsuga, R. Hosokawa, M. Yoshida, Y. Sato, and Y. Akagawa, "A Novel Handy Probe for Tongue Pressure Measurement," Int 7 Prosthodont, vol. 15, no. 4, pp. 385-8, 2002.

[48] A. Hewitt et al., "Standardized Instrument for Lingual Pressure Measurement," Dysphagia, vol. 23, no. 1, pp. 16-25, Mar. 2008, doi: 10.1007/s00455-007-9089-0.

[49] K. Tsuga, M. Maruyama, M. Yoshikawa, M. Yoshida, and Y. Akagawa, "Manometric Evaluation of Oral Function With a Hand-Held Balloon Probe," Journal of oral rehabilitation, vol. 38, no. 9, 2011, doi: 10.1111/J.1365-2842.2011.02202.X.

[50] S. N. Robinovitch, C. Hershler, and D. Romilly, "A Tongue Force Measurement System for the Assessment of Oral-Phase Swallowing Disorders," Arch Phys Med Rehabil, vol. 72, no. 1, pp. 38-42, 1991.

[51] M. Stone and A. Lundberg, "Three-dimensional tongue surface shapes of English consonants and vowels," The fournal of the Acoustical Society of America, vol. 99, no. 6, pp. 3728-37, Jun. 1996, doi: 10.1121/1.414969.

[52] H. Takemoto, "Morphological Analyses of the Human Tongue Musculature for Three-Dimensional Modeling," fournal of Speech, 
Language, and Hearing Research, vol. 44, no. 1, pp. 95-107, Feb. 2001, doi: 10.1044/1092-4388(2001/009).

[53] K. M. Hiiemae and J. B. Palmer, "Tongue movements in feeding and speech," Critical reviews in oral biology and medicine: an official publication of the American Association of Oral Biologists, vol. 14, no. 6, pp. 413-29, 2003, doi: 10.1177/154411130301400604.

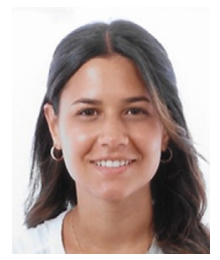

Marta Carlota Díaz Sáez

Marta Carlota Díaz Sáez got her Physical Therapy Degree at the Centro Superior de Estudios Universitarios La Salle (CSEULS), Universidad Autónoma de Madrid (UAM) in 2016. She is a current PhD student by the same university. She also developed a postgraduate study in Physical Therapy for Myofascial Pain, Dry Needling at the CSEULS (UAM) in 2016. She studied an Official University Master of Advanced Physical Therapy in the Treatment of Pain at the CSEULS (UAM) in 2016-2017. She got her expert diploma of Physical Therapy for Temporomandibular Disorders and Headaches at the CSEULS (UAM) in 2020. She is Orthopaedic Physical Therapist in the Instituto de Rehabilitación Funcional de La Salle (IRF La Salle). She is a full professor in Dry Needling postgraduate course at the CSEULS (UAM). She also has been a full professor in Physical Therapy degree in the Bases of Physical Therapy and Electrotherapy subject and in the International Academic Activities (ACAI) program for Erasmus students at the CSEULS (UAM). She is author of 2 scientific articles and her main research area is Craniofacial Pain. She belongs to CranioSpain Research Group at the CSEULS (UAM).

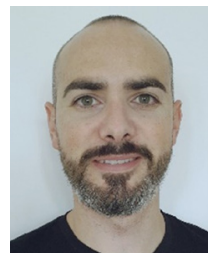

Héctor Beltrán Alacreu

Héctor Beltrán Alacreu got his Physical Therapy Degree at the Universidad Europea de Madrid in 2010. He also studied an Official Master in Orthopedic Manual Therapy at the Universidad Europea de Madrid in 2011 and an Official Master in Study and Treatment of Pain at the Universidad Rey Juan Carlos in 2016. He is a PhD at the Universidad Rey Juan Carlos in 2015. He has 10 years of experience as an assistant physiotherapy. He is a professor and researcher at the Universidad de Castilla-La Mancha in the Faculty of Physiotherapy and Nursing. Finally, he has published more than 30 original scientific articles His main research areas are in Physical Rehabilitation and Musculoskeletal Pain.

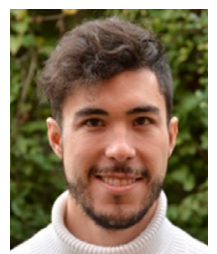

Javier Gil Castillo

Javier Gil Castillo got his BCs degree in Biomedical Engineering in Technical University of Madrid (UPM) in 2016. His Bachelor Thesis at the Biomedical Technology Center (CTB) was focused on the techniques of magnetic hyperthermia and magnetometry. He completed his internship in the innovation unit of the San Carlos Clinical Hospital in Madrid. He also got his MSc degree in Biomedical Engineering in University of Navarra, TECNUN, in 2018. His Master's thesis was developed in the Neural Rehabilitation Group (Cajal Institute, CSIC) and focused on the design, the integration and the implementation of a functional electrical stimulation (FES) assistance system during the gait for patients with incomplete spinal cord injury. He is currently working as a researcher in the Neural Rehabilitation Group since 2018. During the period at CSIC he has published an article in the Journal of NeuroEngineering and Rehabilitation (JNER) and two publications in congresses (IROS and ICNR) and has participated in projects at national and international level as REASISTE and TAILOR. He is currently working on his doctoral thesis related to gait rehabilitation using electrostimulation and wearable robots.

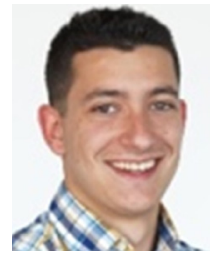

\section{Gonzalo Navarro Fernández}

Gonzalo Navarro Fernández got his Physical Therapy Degree at the Centro Superior de Estudios Universitarios La Salle (CSEULS), Universidad Autónoma de Madrid (UAM) in 2015. He also developed different postgraduate studies in Health Sciences Applied Statistics, in Advanced Methods in Applied Statistics and in Systematic Reviews and Metanalysis at the Universidad Nacional de Estudios a Distancia (UNED). He studied and Official University Master of Advanced Physical Therapy in the treatment of Pain at the CSEULS (UAM) in 20152016. He obtained his expert diploma of Physical Therapy in the Management of temporomandibular disorders and headaches at the CSEULS (UAM) in 2020. $\mathrm{He}$ is currently working in his $\mathrm{PhD}$ at the Universidad Rey Juan Carlos (URJC). He works as orthopaedic physical therapist in the Instituto de Rehabilitación Funcional y Ciencias Aplicadas al Deporte La Salle (IRF La Salle). He also works as full professor in Physical Therapy Degree at CSEULS in different subjects such as Orthopaedic Manual Therapy I, Biomechanics, Physical Therapy assessment and evaluation; and in 2 Physical Therapy postdegrees: Professional Development Course in Invasive Physical Therapy and Expert Course in Physical Therapy in the Management of Temporomandibular Disorders and Headaches. Finally, he belongs to CranioSPain Research Group at the CSEULS (UAM) and is author of 9 scientific articles in his main research area (Cranio-cervico-facial pain) and other physiotherapy areas.

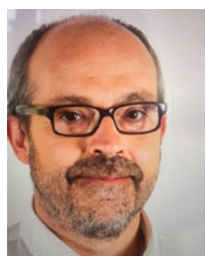

\section{José Luis Cebrián Carretero}

José Luis Cebrián Carretero got his Medical Degree at the Universidad Autónoma de Madrid (UAM) in 1993. He is $\mathrm{PhD}$ by the same university in 2005 . He also studied Oral and Maxillofacial training H.U. La Paz from 1995 to 2000. He got is Dental Degree in the Universidad Europea de Madrid (UEM) in 2009. He is fellow of the European Board of Oral and Maxillofacial Surgery, Specialist in Oral and Maxillofacial Surgery, La Paz University Hospital, Madrid and Chief of Division in Oral and Maxillofacial Surgery, La Paz University Hospital, Madrid, Hospital Sur de Madrid and Clínica La Luz. Finally, he is Vice-President of the SECOM (Spanish Society of Oral and Maxillofacial Surgery). He is author of 2 books, 15 chapters and more than 50 scientific articles. His main research areas are Implantology, Head and Neck Oncologic Surgery and Orthognatic and Oral Surgery.

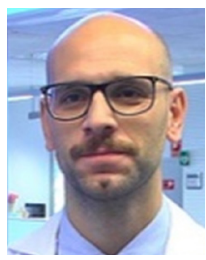

Alfonso Gil Martínez

Alfonso Gil Martínez got his Physical Therapy Degree at the Hospital Militar Gómez Ulla in 2003. He also studied an Official Master in Neuromotor Control at the Universidad Rey Juan Carlos in 2011-2012. He is a PhD by the same university in 2017. He is an assistant physiotherapist with 20 years of experience. He is a professor and researcher at the Hospital Universitario La Paz and Centro Superior de Estudios Universitarios La Salle. Finally, he has published more than 40 original scientific articles in the Pain area, several book chapters and has raised more than 1 million euros in competitive funding for healthcare research and innovation projects. 